\title{
Advantages of Modified Stoppa Approach in Mangement of Acetabular Fractures
}

M.G.Al-Ashhab, H.A.AL-Attar, S.A.Shoulah and M.S.Abd Elkareem

Orthopedic Surgery Dept., Faculty of Medicine, Benha Univ., Benha, Egypt

E-Mail:Salah2356@gmail.com

\begin{abstract}
This study presents our experience of internal fixation of the acetabular fractures and pelvic fractures using a newly modified Stoppa approach, which provides closer visualization to the entire anterior column, quadrilateral surface and the pelvic brim portion of the posterior column, and permits stable internal fixation for the acetabular fracture patterns with main anterior displacement and the anterior and lateral parts of the pelvis. The present study was a prospective clinical study including 10 patients with acetabular fractures who were treated operatively using modified Stoppa approach. The classification of acetabular fractures described by Letournel and Judet. All operative data was recorded Patients were followed-up for 12 months after the operation. The maximum displacement of the bony fragment was measured in the unit of mm by using PACS, Marosis mview ver. 5.4 Our result shows the incidence of perioperative complication. Two patients developed DVT and two patients developed obturator nerve palsy. Our result shows the radiological outcomes of the included patients. The mean CT gap was $1.685 \pm 0.27 \mathrm{~mm}$. Eighty percent of the patients had anatomical Mattaclass. Our result shows the Merle D'Aubigne-Postel score of the included patients. Eighty percent of the patients hadexcellent score. The incidence of MVO after PPCI may be lowered by the addition of manual TA $(6,24)$. The results of the present study show that the additional intracoronary administration of a high dose of adenosine may further improve STR up to 70\%. Future larger studies with clinical endpoints are warranted to confirm the benefit of intracoronary administration of a high dose of adenosine in addition to glycoprotein IIb/ IIIa antagonist administration and TA in the setting of PPCI or rescue PCI.
\end{abstract}

\section{Introduction}

Those anatomic rebuilding of the acetabular joint surface in the medicine from claiming displaced acetabular fractures need been esteemed to a chance to be key if disappointment from claiming joint-preserving surgery will be will a chance to be avoided [1$3]$.

However, anatolian dialect diminishment will be getting to be progressively bulky as those manifestation about fractures need modified (e. G. Impaction of the superomedial acetabular roof), basically Similarly as an aftereffect for a watched expansion On geriatric trauma [2,4 and 6]. A further sway on the result might have been identified with get horribleness [7] which might be about specific imperativeness in the elderly with confined physiological saves.

All assention exists for the utilization of the altered Stoppa methodology for the larger part about fractures that could a chance to be figured out how for a ilioinguinal methodology $[8,9,10]$, Yet debate stays in regards this utility's methodology done All the more was troublesome crack examples for example, such that posterior section fractures.

Therefore, alongside those Stoppa approach, a extra parallel iliac wing window may be important in place should accomplish this surgical errand. Jakob et al. [8] Furthermore additional recently, andersen et al. [11] de $\neg$ scribed An changed methodology utilizing those Stoppa Also illiac surgical window for those medicine of acetabular What's more pelvic ring fractures. This approach got to be popullar over days gone by decade for foremost col-umn fractures Furthermore may be suitableness to those ma $\neg$ jority about cases, same time giving work to fantastic visualization about and entry of the quadri $\neg$ lateral plate and parts of the posterior colᄀumn [12].

This contemplate displays our experience about interior obsession of the acetabular fractures What's more pelvic fractures utilizing An recently altered Stoppa approach, which gives closer visualization of the whole foremost column, quadrilateral surface and the pelvic overflow bit of the posterior column, and permits stable inner obsession to the acetabular crack examples for primary foremost relocation and the foremost What's more parallel parts of the pelvis.

Those point for this proposal is should assess the functional, clinical and radiological Conclusion for medication about acetabular fractures by altered Stoppa approach.

\section{Patient and Method}

The present study was a prospective clinical study including 10 patients with acetabular fractures who were treated operatively using modified Stoppa approach at Banha university hospital during the period from January to December 2019.

We included adult patients with recent fractures of acetabulum of: 
- Anterior wall and columns

- Both columns fracture

- Patients who were medically-fit for surgery

We excluded patients with the following criteria:

- Old neglected fracture.

- Delayed union or non-union fracture

- Fractures associated with neurovascular injuries

- Patients who were not medically fit for surgery

The classification of acetabular Fractures portrayed by Letournel Also Judet may be the regularly utilized arrangement framework. In this system, acetabular fractures would separated under two fundamental groups: basic break sorts and the that's only the tip of the iceberg intricate connected crack sorts. Straightforward break sorts need aid disengaged fractures of you quit offering on that one divider alternately section alongside transverse fractures; this kind incorporates fractures of the posterior wall, posterior column, foremost wall, alternately foremost section Also transverse fractures. Cohorted crack sorts need All the more perplexing crack geometries What's more incorporate T-type fractures, consolidated fractures of the posterior section and wall, consolidated transverse and posterior divider fractures, foremost section fractures with An posterior hemitransverse fracture, and both-column fractures.

Every one agent information might have been recorded. Similarly as quickly Similarly as patients landed toward our crisis service, sufficient revival Furthermore haemodynamic soundness might have been attained. Skeletal footing for distal femur might have been Ordinarily used, Furthermore low sub-atomic weight heparin $(10 \mathrm{mg}$, qd) might have been utilized to counteractive action for profound vein thrombosis. Pelvic X-ray, electronic tomography (CT) examine and threedimensional (3D) reproduction were routinely performed. An doppler ultrasonography of the pelvis and the easier extremities might have been completed will discount At whatever profound venous thrombosis (DVT). Those $3 \mathrm{~d}$ printing engineering might have been utilized within exactly muddled instances to print pelvic model, and the contralateral hemipelvis might have been also mirror-printed will preshape plates on the model. One day When the surgery, schedule enema, urinary catheterization Furthermore arrangements to blood transfusion must all a chance to be reassessed.
The tolerant might have been put recumbent around a radiolucent working table that permits sufficient visualization once AP What's more Judet radiographs. A table that facilitates parallel footing of the hip might have been supportive to eliminating deforming strengths on the foremost section and quadrilateral surface. Those harmed appendage might have been hung free, and the ipsilateral hip Furthermore knee were looked after to slight flexion utilizing footing on the flexed leg or An radiolucent triangle. This position relaxes the iliopsoas muscle and the outside iliac vascular pack. An Foley catheter might have been used to ensure the bladder, enhance visualization, and screen liquid parity. Those specialist remained on the contralateral side on move forward visualization for What's more right of the accurate intrapelvic pit. A head light or An fiber-optic light retractor might have been utilized At necessary.

An Pfannenstiel entry point might have been settled on 1 should $2 \mathrm{~cm}$ better than those pubic symphysis. Analyzation might have been conveyed down through those skin and subcutaneous tissue of the level of the rectus belt. The rectus belt might have been part in line with its fibers, and the transversalis belt might have been chiseled recently better than those pubic symphysis. Distant parallel analyzation might have been demoralized due to those hazard for harm of the spermatic string or round ligament Concerning illustration they retreat the outside ring. Gruff dismemberment of the Retzius space might have been performed. This space might have been stuffed for laparotomy sponges with secure those urinary bladder What's more urethra.

Subperiosteal analyzation might have been performed along the pubis, unrivaled pubic ramus, posterior surface of the ramus, What's more pelvic overflow dependent upon under the inward iliac fossa. An pointed Hohmann retractor might have been set again those pubic tubercle on reflect the rectus musculature. Concerning illustration those analyzation might have been broadened at the acetabulum, An Deaver retractor alternately pliable retractor might have been used to protect Furthermore lift the outside iliac vessels and the iliopsoas muscle. The vascular anastomoses were ligated propel those analyzation more remote along those pelvic overflow Also quadrilateral surface.

Extra introduction might have been gotten with distal development for periosteal dismemberment along the pelvic overflow. Those iliopectineal belt might have been separated In the foremost section and the arch 
of the hip bone socket. An taymyr landmass or Deaver retractor might have been set under those iliopsoas muscle At necessary should ensure the outer iliac vessels. Dismemberment might have been proceeded to those foremost perspective of the sacroiliac joint on uncover those whole pelvic overflow.

Next, the quadrilateral surface and the average part of the posterior section were uncovered. Those obturator neurovascular pack might have been identifier in the fat average of the internal obturator muscle. This pack might have been disengaged What's more ensured with An limited pliable retractor put delicately in the sciatic score alternately the middle of the quadrilateral surface and the interior obturator muscle. Parallel withdrawal of the femoral mind for a trochanteric footing pin might have been accomplished for introduction Also diminishment Toward eliminating deforming strengths on the medially removed break.

Foremost section fractures were accessed utilizing those parallel window of the ilioinguinal methodology. An entry point might have been produced along those iliac peak will uncover the insertion of the angled abdominal muscles. These muscles were discharged to permit analyzation through the peak under those internal iliac fossa. Those iliac muscle might have been raised subperiosteally, exposing the iliac fossa of the pelvic overflow and also the foremost angle of the sacroiliac joint. Assuming that supplement vespula vulgaris foramina were encountered along those internal iliac fossa; An bone wax might have been connected of the foramina should control dying. A Ganz or gruff Hohmann retractor might a chance to be set precisely under those sciatic score or along those quadrilateral surface should move forward visualization. This assistant parallel window facilitates diminishment of secondary foremost section fractures Furthermore placement of posterior section slack screws.

The foremost section might have been decreased through those AIP approach or a parallel window. An pelvic collinear decrease cinch (Synthes), standard pelvic decrease clamp, alternately ballspike pusher (ie, picador) might have been used to decrease the foremost crack. Those decrease might have been settled done standard design for An pelvic reproduction plate for alternately without slack screws along the pelvic overflow Furthermore pubic distinction.

After the wound might have been irrigated and hemostasis might have been achieved, An channel might have been set in the Retzius space. Assuming that those parallel window might have been used, An second channel might have been put in the internal iliac fossa. Those rectus abdominis muscle and the interim the middle of the outside angled muscles and the abductors were shut for absorbable sutures, accompanied by layered Conclusion of the subcutaneous What's more cutaneous tissues.

Drains were uprooted when waste falls The following $25 \mathrm{ml}$ for every 8 hours. Nonintrusive treatment for toe-touch weight bearing might have been accomplished for 12 weeks. Give back about inside Furthermore bladder capacity were precisely monitored. The wound might have been checked 7 should 14 days after release starting with the healing facility. Extra postoperative catch up will be completed toward 6 weeks.

Patients were followed-up for 12 months after those operation. The greatest relocation of the hard part might have been measured in the unit for mm Eventually Tom's perusing utilizing PACS, Marosis mview ver. 5. 4 (Marotech inc. , Seoul, Korea) on the straightforward radiographs and the ct pictures to assessment of the crack diminishment personal satisfaction. Those crack might have been ordered dependent upon those criteria for Matta et al. In this ponder.

- If the measured displacement was less than $0-1 \mathrm{~mm}$, it was categorized as anatomical reduction

- If it was in the range of 2-3 mm, it was categorized as imperfect reduction

- If it was > $3 \mathrm{~mm}$, it was categorized as poor reduction.

In addition, follow-up hip function score was graded by the Merle d'Aubigne and Postel score system according to the sum of score of pain, walk and range of motion:

- Excellent (18 points)

- Good (15-17 points)

- Fair (13-14 points)

- $\quad$ Poor (>13 points).

\section{Results}

Our result shows the demographic and laboratory characteristics of the included patients. The mean age of the included patients was $43.6 \pm 9.3$ years and the majority of them were males $(70 \%)$.

Our result shows the mechanism of injury of the included patients. The most common mechanism of injury was traffic accident $(80 \%)$, followed by fall from height $(20 \%)$.

Our result shows the fractures characteristics of the included patients. The fracture classifications were $\mathrm{AC}+\mathrm{AW}(50 \%)$, both columns $(20 \%)$, and both columns + iliac wing (30\%). The most common associated injury was spine fracture $(50 \%$ 


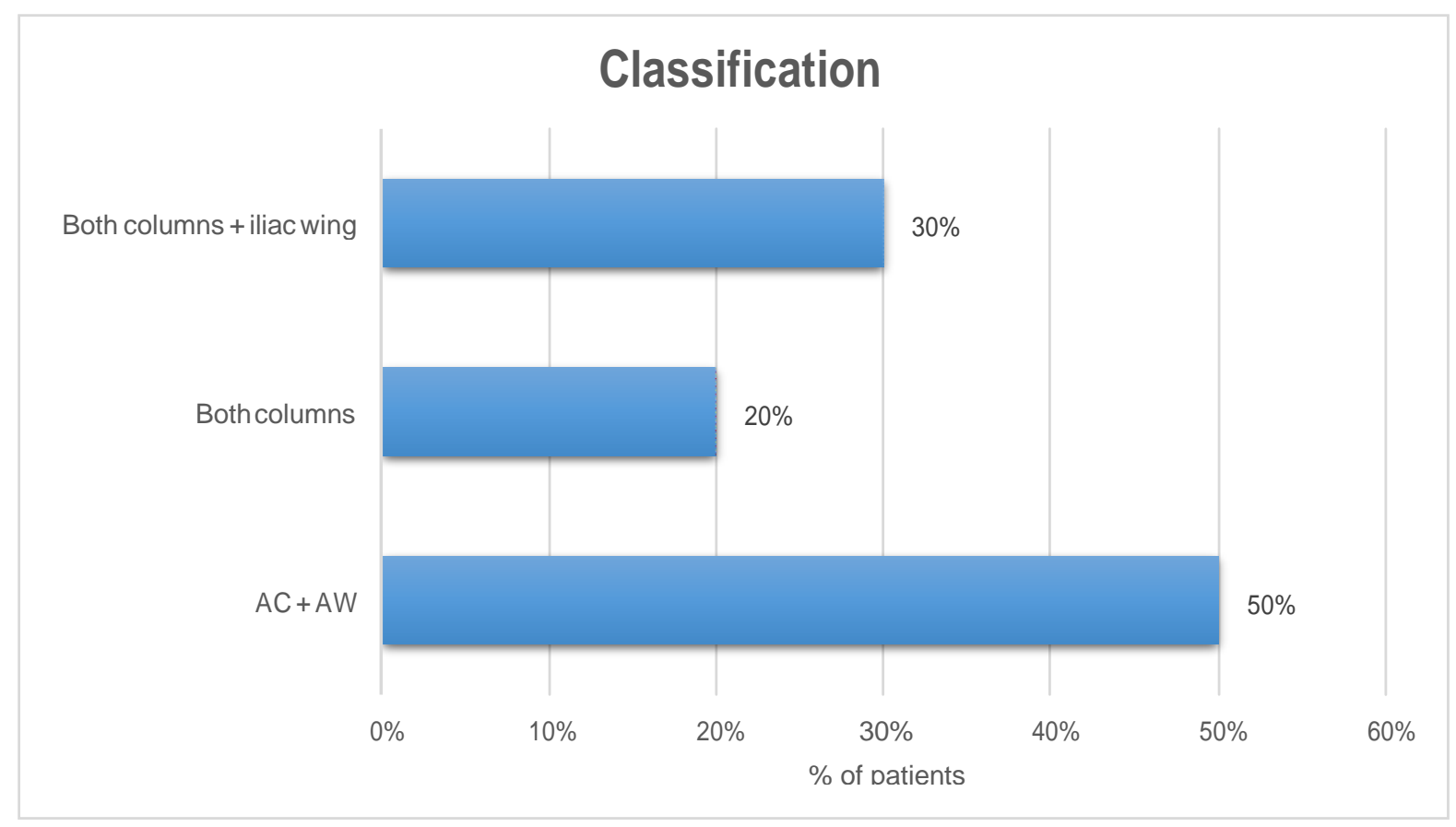

Fig (1) Distribution of Fracture Classification.

Our result shows time to surgery of the included patients. The mean time to surgery of the included patients was $7.90 \pm 1.4$ days.

Our result shows intraoperative characteristics of the included patients. The operative time of the included patients was $216.4 \pm 27.5$ minutes. The mean blood loss was $910.2 \pm 196.7 \mathrm{~mL}$

Our result shows the intraoperative characteristics of the included patients. All patients had iliac window and no patients required additional Kocher-Langenbeck approach
Our result shows the incidence of perioperative complication. Two patients developed DVT and two patients developed obturator nerve palsy.

Our result shows the radiological outcomes of the included patients. The mean CT gap was $1.685 \pm 0.27 \mathrm{~mm}$. Eighty percent of the patients had anatomical Mattaclass.

Our result shows the Merle D'AubignePostel score of the included patients. Eighty percent of the patients hadexcellent score.

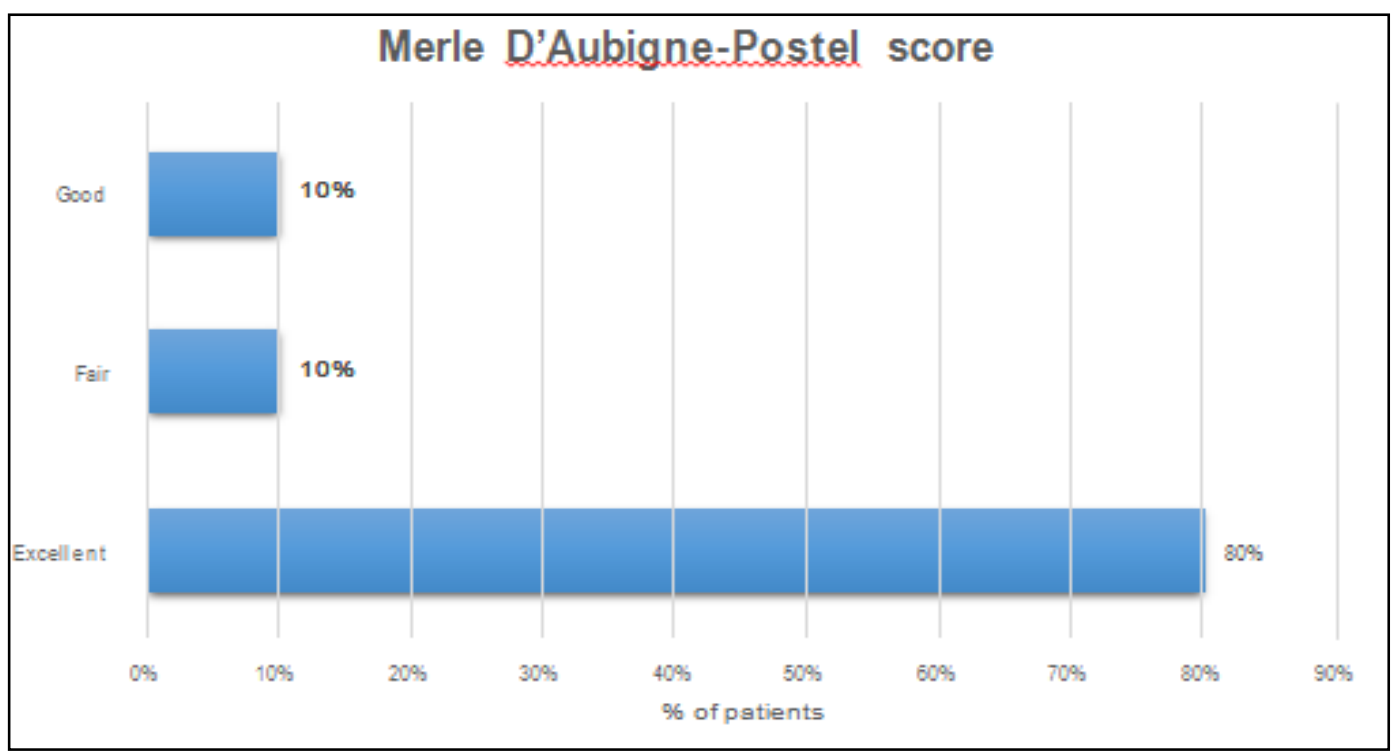

Fig (2) Merle D'Aubigne-Posel score 


\section{Discussion}

Acetabular fractures are Around the The greater part intricate wounds approached Toward orthopedic surgeons. Those initial known portrayals from claiming surgical obsession of acetabular fractures were instance reports for 1943. Over 1964, robert Judet, jean Judet, Also Émile Letournel distributed An point of interest article describing a arrangement framework What's more surgical methodologies should treat acetabular fractures. These teachings required a critical impact on clinical results then afterward surgical obsession of acetabular fractures [13].

Different methodologies need aid great depicted in the written works Furthermore incorporate the Kocher-Langenbeck, iliofemoral, ilioinguinal, joined together foremost and posterior approaches, developed iliofemoral, transtrochanteric, Furthermore triradiate methodologies. Alongside those advancement for minimally obtrusive systems meant toward minimizing surgical dissection, endeavors bring been made with treat acetabular fractures with a much lesquerella extensile approach [14].

Stoppa depicted as much approach Initially over 1973 as An subperitoneal average approach for the medicine for groin hernias for the help of a dacron tulle prosthesis. Done 1994, cole Also Bolhofner portrayed those Stoppa approach, administering the specialist should stand In the inverse side of the included hip joint Throughout diminishment and obsession of the acetabular fractures, permitting to regulate visualization of the average wall, dome, quadrilateral plate, Also extending Concerning illustration a long way Similarly as those sacroiliac joint. However, this methodology doesn't empower maneuvering the fractures to anatomic decrease of the iliac wing, An vital venture in anatolian dialect rebuilding of the foremost wall [15].

Therefore, alongside those Stoppa approach, a extra parallel iliac wing window may be vital so as with accomplish this surgical assignment. Jakob et al. Also more recently, portrayed An changed approach utilizing those Stoppa Also illiac surgical window for the medication of acetabular and pelvic ring fractures. This methodology got to be mainstream through as long as decade for foremost section fractures Also may be suitableness to the greater part from claiming cases, same time giving work to fantastic visualization about and right of the quadrilateral plate Furthermore parts of the posterior section [11].
However, there will be An shortage in the distributed writing viewing the conclusions about Stoppa methodology. Thus, we led the introduce investigation so as on assess the results of altered Stoppa methodology to patients with foremost wall, column, or both section acetabular fractures.

Those introduce consider might have been a clinical investigation that might have been directed around An successive companion about 10 patients for acetabular fractures who were dealt with operatively utilizing altered Stoppa methodology during Banha college healing center Throughout the period starting with january will december 2019 .

The the study of disease transmission from claiming these fractures in the usa Furthermore europe need been great established, demonstrating to tantamount bimodal period circulations (ie, the recurrence about fractures happens done two agdistis peaks: those primary top speaking to adolescent patients manage high-thickness lipoprotein fractures and the second top more seasoned patients manage low-energy osteoporotic/fragility fractures) [16].

In the exhibit study, those mean agdistis of the incorporated patients might have been 43 . $6 \pm 9.3 \mathrm{~A}$ long time and the lion's share about them were guys $(70 \%)$.

Similarly, [16] concentrated information from trauma databases for patients admitted with acetabular fractures figured out how surgically starting with over 2,800 doctor look assignments led from April 1, 2009 to March 31, 2010 should 2012 to you quit offering on that one chinese focus Furthermore from 2008 to 2012 for particular case us focal point. Intend agdistis during occasion when of damage might have been different between china and the USA, toward 40 quite some time with a unimodal circulation What's more 44 quite some time with a bimodal conveyance. Those greater part of patients were guys.

Engine vehicle mishaps would those mossycup oak normal reason for acetabular fractures and the kind about crack need been indicated should associate for course about impact [17].

Those The majority normal component for harm in the available consider might have been movement mischance (80\%), emulated Eventually Tom's perusing fall from stature $(20 \%)$.

In understanding for our findings, [17] meant with assess those epidemiological profile for AF over france may be consonant for information starting with european caseseries investigations. Every one patients 
figured out how to AF between over 2,800 doctor look assignments led from April 1, 2009 to March 31, 2010 What's more 2014 were included in this single-centre review consider. The middle of over 2,800 doctor look assignments led from April 1, 2009 to March 31, 2010 Furthermore 2014, 414 patients were admitted for AF. Mean period might have been 49. 4 A long time (range: $15-101$ years). There were $162(70 \%)$ guys. The mossycup oak normal instrument for harm might have been movement mischance.

Acetabular fractures are regularly secondary vitality Also thusly frequently all the introduce Previously, blending with different organ wounds. In the display study, $70 \%$ of the patients required connected damages the mossycup oak as a relatable point copartnered damage might have been spine crack $(50 \%)$.

Likewise, [18] retrospectively broke down 126 hip bone socket fractures that needed been treated surgically during under the middle of Walk 2006 Furthermore november 2008. 76. $8 \%$ were male; the imply agdistis might have been 39. 6 quite some time. Those trauma system might have been movement mishaps over $59 \% ; 70 \%$ of the patients exhibited cohorted fractures.

The results of precisely treated acetabular cracks are reliant on numerous variables. Radiological appraisal utilizing the Matta scoring framework deciphers "amazing" for an ordinary seeming hip joint, "great" for gentle changes with negligible sclerosis and joint narrowing $(<1 \mathrm{~mm})$, "reasonable" for middle changes with moderate sclerosis and joint narrowing $(<50 \%)$, and "poor" for cutting edge changes 19).

In the current examination, we found that the mean CT hole was $1.685 \pm 0.27 \mathrm{~mm}$. $80 \%$ of the patients had anatomical Matta class.

In concordance with our discoveries, Andersen and associates [11] intended to measure the decrease of acetabular cracks with relocation of the front and back segment by utilizing just a solitary foremost methodology that consolidates the altered Stoppa approach with the parallel window of the exemplary ilioinguinal approach. A back to back associate of 17 patients with uprooted acetabular cracks treated operatively with utilization of just a front methodology that consolidated the altered Stoppa approach with the horizontal window of the ilioinguinal approach. Anatomic decrease of the articular surface was acquired in $14(82 \%)$ patients, flawed radiographic decrease in three $(18 \%)$, and poor radiographic decrease in none $(0 \%)$.
Thus, [15] introduced their involvement with utilizing the Stoppa approach. In a 5-year audit of 60 acetabular breaks that experienced open decrease and inward obsession utilizing the altered Stoppa approach. Postoperative radiological assessment uncovered anatomical decrease in $54 \%$ of the patients, agreeable in $43 \%$, and unsuitable in $3 \%$ of the patients. In general, there were 15 minor and significant inconveniences

[20] assessed brings about patients with hip bone socket cracks with the adjusted Stoppa approach. Between September 2008 and August 2012, 289 patients with acetabular breaks were treated at our Level I emergency room. Radiographic reviewing of break decreases after medical procedure uncovered that $27(75 \%)$ were anatomic, six $(17 \%)$ were good, and three $(8 \%)$ were inadmissible. A sum of $94 \%$ of the breaks joined together.

The Modified Merle d'Aubigné and Postel Method instrument is utilized by different creators in the pre-and postoperative clinical assessment, since it is considered effectively reasonable and offers basic application.10-17 According to Gonçalves, the boundaries set up in the strategy altered by d'Aubigné and Postel are considered the most handy in the assessment of the hip influenced by illness. The related record of torment, walk and portability in the pre-and postoperative periods checks the treatment results. It is underscored that deficient records prompt blunders in the last assessment, as the outcome is subject to the similar examination. The constant use and the obtained understanding of this instrument have expanded fulfillment in its appropriateness in study protocols [21].

In the current investigation, $80 \%$ of the patients had incredible score as per Modified Merle d'Aubigné and Postel Method.

Y. Yang et al., [22] examined the adequacy of adjusted Stoppa approach with average divider spring plate (MWSP) for including quadrilateral of hip bone socket break. Between March 2008 and September 2013, 38 patients with including quadrilateral of hip bone socket crack were dealt with. At 1 year after activity, the outcomes were amazing in 9 cases, great in 21 cases, general in 5 cases, and poor in 3 cases, and the phenomenal and great rate was $78.95 \%$ as indicated by the Merled' Aubigne and Postel hip score norms.

As to the frequency of perioperative confusions in the current investigation, two patients created DVT and two patients created obturator nerve paralysis.

In concordance with our discoveries, [23] detailed 17 patients with a mean follow-up of 3.2 years. Ten patients were worked by means 
of careful hip disengagement, two patients with a Stoppa approach, and five utilizing a joined or elective methodology. Complexities contained one obturator nerve paralysis in patients with Stoppa approach.

\section{By and large, we can reason that the upside of Stoppa approach are:}

- Relatively easy anatomical dissection in comparison to the ilioinguinal approach

- Offers the surgeon better visibility than the classic ilioinguinal approach of all anterior elements, especially the quadrilateral plate

- Direct control over the corona mortis

- Direct visualization and approach to the posterior column

- Enables fixation and medial buttressing of the quadrilateral plate with a reconstruction plate, placed directly and medially to the hip joint, this has not been previously described in the ilioinguinal approach

\section{References}

[1] N. Briffa, R. Pearce, A. M. Hill, Outcomes of acetabular fracture fixation with ten years' follow-up. The J., bone and joint surgery. British volume, Vol.93(2), PP.229-236, 2011.

[2] T. A. Ferguson, R. Patel, M. Bhandari, Fractures of the acetabulum in patients aged 60 years and older: an epidemiological and radiological study. The J., bone and joint surgery. British volume, Vol.92(2), PP.250257,2010.

[3] Tannast, Moritz, Soheil Najibi, "Two to twenty-year survivorship of the hip in 810 patients with operatively treated acetabular fractures.", Vol.17, PP.15591567,2012

[4] Anglen, Jeffrey , "The "Gull Sign": a harbinger of failure for internal fixation of geriatric acetabular fractures." J., orthopaedic trauma ,vol ,17. 9 ,pp,625$634,2003$.

[5] T. A. Ferguson, R.Patel, M.Bhandari, Fractures of the acetabulum in patients aged 60 years and older: an epidemiological and radiological study. The J., bone and joint surgery. British volume,Vol.92(2), PP. 250-257. 2010.

[6] P. M. Rommens, I P.ngelfinger, Nowak, T. E., Kuhn, S., \& Hessmann, M. H. (). Traumatic damage to the cartilage influences outcome of anatomically reduced acetabular fractures: a mediumterm

retrospective analysis. Injury, vol42(10), pp10431048,2011.

[7] P. V. Giannoudis, M. R. W.Grotz, , C. Papakostidis, , \& Dinopoulos, H. Operative treatment of displaced fractures of the acetabulum: a meta-analysis. The J., bone and joint surgery. British volume, vol,87(1), PP.2-92005.

[8] M. Jakob, R. Droeser, R. Zobrist, , A less invasive anterior intrapelvic approach for the treatment of acetabular fractures and pelvic ring injuries. J., Trauma and Acute Care Surgery,Vol. , Vol.60(6),PP. 13641370.2013

[9] [9] M. A. Karunakar , pp, , T. T. Le, M. J. Bosse,. The modified ilioinguinal approach. J., orthopaedic trauma, Vol.18(6), PP.379-383. 2004

[10] Y. R. Farid, The subinguinal retroperitoneal approach for fractures of the acetabulum: a modified ilioinguinal approach. J., orthopaedic trauma, Vol.22(4), PP.270-275,2008.

[11]R. C.A ndersen, R.V.O'Toole, J. W.Nascone, , Modified stoppa approach for acetabular fractures with anterior and posterior column displacement: quantification of radiographic reduction and analysis of interobserver variability. J., orthopaedic trauma, vol,24(5), PP.271-278, 2010.

[12] P. Guy, M. Al-Otaibi, E. J. Harvey, The 'safe zone'for extra-articular screw placement during intra-pelvic acetabular surgery. J., orthopaedic trauma, Vol.24(5), PP. 279-283, 2010.

[13] N.Ziran , G.L.S.Soles , J.M.Matta . Outcomes after surgical treatment of acetabular fractures: A review. Patient Saf Surg,vol,13,pp, 732019.

[14]P.V. Giannoudis, N.K. Kanakaris , Acetabular fractures. Trauma Orthop. Classif. A Compr. Overv., SpringerVerlag London Ltd, Vol.25(5),PP. 28592,2015.

[15] A. Khoury, Y. Weill, R. Mosheiff , The Stoppa approach for acetabular fracture. Oper Orthop Traumatol , Vol.24,PP.43948.2012.

[16] C. Mauffrey, J. Hao, D.O Cuellar, The Epidemiology and Injury Patterns of Acetabular Fractures: Are the USA and China Comparable? Clin Orthop Relat Res,Vol.472,PP.3332-7,2014.

[17] M. Boudissa, F. Francony, G. Kerschbaumer, Epidemiology and treatment of acetabular fractures in a level-1 trauma centre: Retrospective study of 414 patients over 10 years. Orthop Traumatol Surg Res,Vol.103,PP.335- 
9,2017.

[18] Dias MVF, Goldsztajn F, Guimarães JM, Grizendi JA, Correia M, Rocha TH. EPIDEMIOLOGY OF ACETABULUM Fractures Treated At The Instituto Nacional De Traumatologia E Ortopedia (INTO). Rev Bras Ortop (English Ed,vol,45, PP.474-7,2010.

[19] U.K. Meena, S.K. Tripathy, R.K. Sen , Predictors of postoperative outcome for acetabular fractures. Orthop Traumatol Surg Res, Vol.99,PP.929-35,2013.

[20] M.J. Isaacson, B.C. Taylor, B.G. French, Treatment of Acetabulum Fractures Through the Modified Stoppa Approach: Strategies and Outcomes. Clin Orthop Relat Res,Vol.472,PP.3345-52,2014..
[21] F.K. Ugino, C.M. Righetti, D.P.L. Alves, Evaluation of the reliability of the modified merle d'aubigné and postel method. Acta Ortop Bras,Vol.20,PP.2137,2012.

[22] Y. Yang, J . Yue, P .Wen , MODIFIED Stoppa Approach With Medial Wall Spring Plate For Involving Quadrilateral Of Acetabulum Fracture. Zhongguo Xiu Fu Chong Jian Wai Ke Za Zhi, Vol.29, PP.270-4,2015.

[23] M. Tannast, K.A. Siebenrock , Die operative Behandlung der Azetabulum-TFraktur über eine chirurgische Hüftluxation oder einen Stoppa-Zugang. Oper Orthop Traumatol , Vol.21, PP.25169,2009 . 